\section{Bericht vom Welt Thrombose Tag 2021}

In diesem Jahr geht es um Patientenaufklärung und Stärkung der eigenen Gesundheitsvorsorge. Der Bericht wirft viele Fragen auf und fordert uns als Gesellschaft heraus, aktiv an der Lösung mitzuarbeiten!

\section{Gesundheitskompetenz: So wichtig wie gesunde Ernährung und Bewegung}

Rund 2/3 der Deutschen verfügen über eine unzureichende Gesundheitskompetenz. Fakt ist auch: Google behindert die Auffindbarkeit valider Gesundheitsinformationen. Der Arzt ist immer noch der wichtigste Ansprechpartner in Gesundheitsfragen.

Berlin, 13. Oktober 2021 - Informationsangebote zu Gesundheitsthemen stehen heute in einem bisher ungekannten Ausmaß im Netz und auf Social-Media-Kanälen allen zur Verfügung. Gleichzeitig zeigen Studien, dass immer weniger Menschen sich in diesem Angebotsdickicht zurechtfinden und valide Informationen von nichtvaliden nicht unterscheiden können. Das Aktionsbündnis Thrombose hat deswegen zum 8. WeltThrombose-Tag - dem 200. Geburtstag Rudolf Virchows - am 13. Oktober 2021 eine Podiumsdiskussion zum Thema: „Patienten-Empowerment: Patienten stark machen mit validen Informationen!“ veranstaltet.

Ist dies tatsächlich eines unserer vordringlichsten Probleme in unserem Gesundheitssystem? Ja! Denn mehr Gesundheitskompetenz heißt zugleich auch ein Mehr an Gesundheit für die Menschen, weniger Notdiensteinsätze, weniger Krankenhausaufenthalte und Arztbesuche - dies ist nur ein Ergebnis der Expertenrunde.

Prof. Rupert Bauersachs, Wissenschaftlicher Leiter des Aktionsbündnisses Thrombose, weist in seiner Begrüßung auf die enormen Verbesserungen in der Thrombosetherapie hin: eine leicht zugängliche Diagnostik, zuverlässige und wirksame Therapieoptionen sowie wichtige Erkenntnisse zur Thromboseprophylaxe. Dieses Wissen gehöre in die Bevölkerung vermittelt und könnte Leben retten.

\section{Präventionsthemen werden besonders schlecht verstanden}

Die Gründe, warum diese Erkenntnisse nicht zur Anwendung kommen, liefert Prof. Dr. Doris Schaeffer, Fakultät Gesundheitswissenschaften der Universität Bielefeld. Aktuelle Zahlen aus dem 2. Health Literacy Survey Deutschland zeigen: Nahezu $60 \%$ der Deutschen haben demnach Schwierigkeiten, Gesundheitsinformationen zu finden, zu verstehen, zu beurteilen und anzuwenden. Besonders betroffen sind Menschen mit niedriger Bildung, niedrigem Sozialstatus und ab dem Lebensalter 65 plus. Bemerkenswert dabei ist, dass Informationen zur Gesundheitsförderung und Prävention als am schwierigsten eingeschätzt werden.

Was braucht es jedoch, um gute Gesundheitsinformationen bereitzustellen? Jens Richter, Chefredakteur des Patientenportals NetDoktor, betont die Rolle des Wissensvermittlers, der die Bedürfnisse der Patienten gut kennt, um diese Inhalte in Suchanfragen zu übersetzen, die dann auch beantwortet werden können. Dabei, so Richter, sind die Grenzen zu evidenzbasierten Inhalten gegebenenfalls auch zu verlassen. Gerade die Corona-Pandemie habe gezeigt, dass viele Anfragen nicht auf evidenzbasierte Fragestellungen abheben.

Hannelore Loskill, Bundesvorsitzende der BAG Selbsthilfe, mahnt die Aktualität von Informationen an. „Nach Informationen suchen, das tun Kranke, und die müssen die richtigen Informationen bekommen. Es ist eben nicht der gesunde 30-jährige Versicherte, der nach Informationen sucht." Große Probleme habe es beispielsweise zu Beginn der Pandemie gegeben, leichte Sprache und gebärdensprachliche Informationen in die verschiedenen digitalen Kanäle zu bekommen. Kernzielgruppe von Gesundheitsinformationen seien nach wie vor die Betroffenen.

\section{Google entscheidet über die Relevanz von Gesundheitsinformationen}

Ein grundsätzliches Problem der Informationsvermittlung sieht Dr. Klaus Koch, Chefredakteur von gesundheitsinformation.de: „Wir haben einerseits inzwischen eine Menge an Informationen in der Breite und Tiefe auf gesundheitsinformation.de bereitgestellt; andererseits stellt sich die Frage, wie der eine Mensch nun gerade die Frage, die er hat, beantwortet bekommt. " Alle digitalen Internetangebote befinden sich in der Situation, dass sie über Google über $90 \%$ der User zugewiesen bekommen. „Google entscheidet, was gesehen wird und was nicht gesehen wird. Google entscheidet, was oben auf der Trefferliste landet und was unten auf der Trefferliste erscheint. Wir können es Google nicht überlassen, was gute und was schlechte Gesundheitsinformationen sind. Wie bekommen wir die guten Informationen an die Menschen vermittelt? Da wende ich mich auch an die Ärzte. Wir brauchen da eine Arbeitsteilung“, so Koch.

Dr. Robert Eisele bemerkt die Verunsicherung der Patienten durch widersprüchliche Informationen. „Die Patienten unterscheiden nicht zwischen guten und nicht guten Informationen, sie registrieren die Widersprüche." Jeder vierte Patient in seiner Praxis sei außergewöhnlich gut informiert. Das Vertrauen der Patienten in die Vermittlungskompetenz des Arztes sei dabei sehr groß. Was die Qualität von Gesundheitsinformationen angehe, so reiche es eben nicht, einen medizinischen Lehrbuchtext zu übersetzen und ins Internet zu stellen. „Die Informationen gehören aufgearbeitet und interpretiert, anhand aktueller Studienergebnisse auch noch einmal rückversichert. Und komplizierte Sachverhalte muss man auch so darstellen, abweichende Meinungen gelten lassen und begründen“, so Eisele.

„Es ist gar nicht so leicht für diese Mediengattung, suchmaschinengetriebene Gesundheitsportale, Präventionsthemen, im Sinne von Awareness-Bildung, zu betreiben“, erklärt Jens Richter. Die meisten Men- 
schen gingen anlassbezogen auf Gesundheitsportale. „Das Suchverhalten zum Thema Thrombose ist sehr stark davon getrieben, ob ich eine Thrombose habe“, so Richter. Vergleiche man die Inzidenz der Thrombose mit dem Suchvolumen zur Thrombose, so sei das Suchvolumen mehr als 10-mal so hoch. Die Menschen gingen aber auch nicht aus dem Antrieb heraus ins Internet, um sich zu informieren, wie eine Thrombose verringert werden könne.

\section{Patienteninformationen sind zu wenig patientenorientiert}

Prof. Gerd Gigerenzer berichtet von einer internationalen Studie, wonach deutsche Frauen den Nutzen von MammografieScreenings am stärksten überschätzen. Ein Land in Europa weise signifikant andere Ergebnisse auf: Frauen im europäischen Teil von Russland schätzten den Nutzen am realistischsten ein, weil ihnen weniger - auch irreführende - Informationen zur Verfügung stünden. „Wir leben in einer Gesellschaft in Deutschland, wo man nicht den Mut und auch nicht das Interesse hat, Frauen so zu informieren, dass sie eine informierte Entscheidung treffen können.“
Die Patienteninformationen zum Mammografie-Screening in Deutschland seien in der Regel wenig patientenorientiert, kritisiert Gigerenzer. „Zu lang, unverständlich und sie geben das, was für die Patientinnen wichtig sei, nicht wieder. „Deswegen haben wir in unserem Harding Center für Risikokompetenz Faktenboxen entwickelt, die über den Nutzen und Schaden in Zahlen informieren. Das sind Informationen, die kann jeder verstehen."

Gesundheitsinformationen würden von Experten gemacht, die entscheiden, was $\mathrm{Pa}$ tienten über bestimmte Krankheiten wissen sollten. Diese Informationen folgten nicht der Perspektive der Rezipienten, gibt Schaeffer zu Bedenken. Die Nutzer müssten mehr in die Ausgestaltung der Informationen einbezogen werden. Darüber hinaus sei der Arzt nach wie vor die prioritäre Anlaufstelle für Gesundheitsinformationen. Die ärztliche Qualifikation hinke diesen Ansprüchen jedoch hinterher und sie würde in der reformierten Ärztlichen Approbationsordnung wieder nicht berücksichtigt. Hannelore Loskill empfiehlt, Patienten durch Fokusgruppen stärker an der Informationserstellung zu beteiligen.
Prof. Gigerenzer sieht dagegen den Gesetzgeber in der Pflicht, für ehrliche, vollständige und verständliche Gesundheitsinformationen zu sorgen. Ein nationales Portal mit evidenzbasierten Gesundheitsinformationen müsse entsprechend beworben werden. Jens Richter widerspricht: „Gesundheitsinformationen können in den Suchmaschinen nicht einfach regulatorisch hochgerankt werden. Das entspricht nicht der Funktionsweise des Google-Algorithmus. Die mittlerweile unaufgeräumten Suchergebnisseiten von Google machen es den Internetnutzern immer schwerer, die Suchergebnisse zu durchdringen und Werbung zu erkennen. “ Die Qualität eines Gesundheitsportals sei daran zu bemessen, dass möglichst viele Suchanfragen beantwortet werden können.

Dr. Jutta Schimmelpfennig, Ingrid Mühlnikel

IMPRESSUM

Verantwortlich für Mitteilungen der DGP:

Dr. med. Erika Mendoza 